\title{
2016 Wilderness E Environmental Medicine Peer Reviewers
}

The Editors express their sincere appreciation to the following individuals who faithfully and skillfully performed peer reviews in 2016 for articles submitted to Wilderness \& Environmental Medicine. WEM continues to have a growing impact on the scientific community. We acknowledge the essential role our peer reviewers play in ensuring the merit and quality of the manuscripts we publish. Many of these individuals reviewed multiple papers, and some also serve on the Editorial Board and maintain Section Editor duties. Without their service we could not produce this high-quality medical journal. (Note: * indicates WEM Editorial Board membership.)

John Anderson, USA

Eldon Askew, USA

Paul S. Auerbach, USA

Howard Backer, USA*

Howard Barkan, USA

Peter Bartsch, Germany

Buddha Basnyat, Nepal*

Benjamin Bassin, USA

Brad L. Bennett, USA*

Michael Bergeron, USA

Jeffrey Bernstein, USA

Cindy Carol Bitter, USA

Michael Bogdasarian, USA

Adolfo Borges, Bolivarian

Republic of Venezuela

Gemma Claire Lewis

Bornick, USA

Carlos E. Bozzini, Argentina

Julio Brito, Chile

Monika Maria Brodmann

Maeder, Switzerland

Hermann Brugger, Italy

Martin Burtscher, Austria

Frank K. Butler, USA

Gabriel Cade, USA

Aaron D. Campbell, USA

Michael Cardwell, USA

Jim Caruso, USA

Edward R. Chu, Australia

John Robert Clarke, USA

Anita T. Cote, Canada

Cord Cunningham, USA

Tracy Alexandra Cushing, USA*

Michael Dacre, USA

Luc De Haro, France

Juan Del Coso, Spain

Thomas Deloughery, USA

Robert W. Derlet, USA

James H. Diaz, USA
Warren C. Dorlac, USA

Brendon Drew, USA

Charles Dumke, USA

Ola Halina Dunin-Bell, Canada

Bruno Durrer, Switzerland*

David Eaton, Canada

Bruce Edgerly, USA

Kurt Power Eifling, USA

Timothy B. Erickson, USA*

Preston J. Fedor, USA

Joshua Fenderson, USA

Joseph Derek Forrester, USA

Hin Tat T. Fung, China

Nathalie Garcia-Russell, USA

Flavio G. Gaudio, USA

Charles J. Gerardo, USA

Stephen D. Giebner, USA

Milton Glatterer, USA

Todd Golden, USA

Torrey Lisa Goodman, USA

Gudmund Grønhaug, Norway

Colin K. Grissom, USA*

Tracy Beth Høeg, USA

Peter Hackett, USA*

Vidal Haddad Junior, Brazil

Charlotte Haldane, UK

N. Stuart Harris, USA

Tatiana Havryliuk, USA

Seth C. Hawkins, USA

Alana Hawley, USA

Carlton Heine, USA

Mary Josephine Hessert, USA

David Hillebrandt, UK

Alastair Hodges, Canada

John Holcomb, USA

Christopher Holstege, USA

Benjamin Honigman, USA

Stephanie Howe, USA

Stephen Hyslop, Brazil
Chris Imray, UK

Hillary Irons, USA

Arthur (Tony) Anthony

Islas, USA

Ahmad Khaldun Ismail, Malaysia

Wills P. Janardanan, India

Dominique Jean, France

James Jeng, USA

Barbara Jones, USA

Noah Jordahl, USA

Jeremy D. Joslin, USA

Nicholas Kanaan, USA

Lee Kaplan, USA

Bengt Kayser, Switzerland

Robert W. Kenefick, USA*

Daniel Keyler, USA

Morteza Khodaee, USA

Kenneth W. Kizer, USA*

Joseph Guy Kotora, USA

Russ Steven Kotwal, USA

Brian Krabak, USA

John F. Kragh, USA

Senanayaka Am Kularatne,

Sri Lanka

Ricky Langley, USA

Wolfgang Lederer, Austria

Jay Lemery, USA*

Brian Andrew Lentz, USA

Justin Christopher Lewis, USA

James Lieberman, USA

Grant S. Lipman, USA*

Lanny Littlejohn, USA

Adam Lund, Canada

Yongjun Luo, China

Robert L. Mabry, USA

Darryl Macias, USA*

Ioannis Mavridis, Greece

Loui McCurley, USA

Laura McGladrey, USA 
Scott Ellis McIntosh, USA

Christine Mermier, USA

John Meyers, USA

James Sibree Milledge, UK

Richard Moon, USA

Roger B. Mortimer, USA

Thomas Myers, USA

Andrew T. Nathanson, USA

Maniraj Neupane, Nepal

Matias Nochetto, USA

Robert Lee Norris, USA

Andrew Nyberg, USA

Gerald O'Malley, USA

Iekhsan Othman, Malaysia

Edward Joseph Otten, USA*

Peter Paal, UK

Andrew Padula, Australia

Prativa Pandey, Nepal

Ryan Paterson, USA

Andre Michael Pennardt, USA

Erica Perrier, USA

Dan D. Petersen, USA

David Pomeranz, USA

Jose Antonio Prieto, Spain

Matiram Pun, Nepal*

Robert H. Quinn, USA*

Jeremiah Ray, USA

Oliver Reisten, Switzerland

Peter Rhee, USA

Jean Paul Richalet, France

Marc Robins, USA
George W. Rodway, USA*

Ian Rogers, Australia

Terry Rolan, USA

Jens Rothenberger, Germany

Brent Ruby, USA

Emily Sagalyn, USA

John R. Saucier, USA

Tim Saunders, UK

Volker Schöffl, Germany*

Michael Schaer, USA

Bernd Volker Scheer, Spain

Andrew Schmidt, USA

Corinne Schmitt, France

Robert Schoene, USA*

Walter Schrading, USA

Richard H. Schreckengaust, USA

Juerg Schweizer, Switzerland

William Selde, USA

Katherine Shea, USA

Suzanne M. Shepherd, USA

David R. Shlim, USA

Jessica J. Slim, USA

William R. Smith, USA

Susanne J. Spano, USA

Swati Srivastava, India

Cynthia B. Stevens, USA

Cris Stickley, USA

Suzy Stokes, UK

Giacomo Strapazzon, Italy

Michael B. Strauss, USA

Kingman Perkins Strohl, USA
Kristin Stuempfle, USA

Douglas Sward, USA

Erik R. Swenson, USA

Choo Hock Tan, Malaysia

David Tan, USA

Nelson Tang, USA

Christopher Tedeschi, USA

Christopher Van Tilburg, USA

Arpana Vibhuti, India

Julian Villar, USA

Jenny Theresa Visser,

New Zealand

Dale R. Wagner, USA

Beat Walpoth, Switzerland

Jonathon Webber, New Zealand

Ian Wedmore, USA

Jacob Andreas Wegelin, USA

Lori Weichenthal, USA

David Wharton, USA

Albert R. Wheeler, USA

Jenna Marie Bartz White, USA

Buddhika T. B. Wijerathne,

Sri Lanka

Peter Wilmshurst, UK

Matthew Wilson, USA

Gordon H. Worley, USA

Quanfu Xue, China

Cedric M. Yoshimoto, USA

David Young, USA

Ken Zafren, USA*

Keith William Zirkle, USA 